\title{
Atypical Case of Chronic Granulomatous Disease: A Case Report
}

\section{Wilian Luan Pilatti Sant'Ana ${ }^{1}$, Bruno Martini de Azevedo ${ }^{1 *}$ and Ney Noronha Raffin ${ }^{2}$}

${ }^{1}$ Department of Medicine, University of Vale do Taquari Medical School, Brazil

${ }^{2}$ Department of Allergy and Immunology, University of Vale do Taquari Medical School, Brazil

${ }^{*}$ Corresponding author: Bruno Martini de Azevedo, Medical students, Department of Medicine, University of Vale do Taquari Medical School, Brazil, Tel: 5554996406202; E-mail: bruno.azevedo@universo.univates.br

Received date: May 15, 2020; Accepted date: May 21, 2020; Published date: May 28, 2020

Citation: Pilatti Sant'Ana WL, Martini de Azevedo B, Noronha Raffin N (2020) Atypical Case of Chronic Granulomatous Disease: A Case Report. Arch Med Vol. 12 Iss.3:10

Copyright: C2020 Pilatti Sant'Ana WL, et al. This is an open-access article distributed under the terms of the Creative Commons Attribution License, which permits unrestricted use, distribution, and reproduction in any medium, provided the original author and source are credited.

\section{Abstract}

Background: Chronic Granulomatous Disease (CGD) is a heterogeneous clinical presentation immunodeficiency, whose origin can be autosomal recessive or X-linked. In this pathology there is a deficiency in the effector mechanisms of phagocytes.

Case study: A review of the patient's medical records was performed, as well as the currently available bibliography. The case report presented is of an 8-year-old male patient, who had a history of recurrent respiratory infections and ICU admissions since 3 years old, being diagnosed with atypical presentation of CGD at 5 years old, and managed with standard prophylactic treatment based on sulfamethoxazole-trimethoprim (SMX-TMP).

Conclusion: CGD has a wide range of symptoms, so treatment should be guided based on each patient. In the presented case, the patient is being treated with a prophylactic combination of Itraconazole and SMX-TMP. Besides, we are still awaiting judicial clearance by the Brazilian National Health System (SUS) for financial support for IFN-gamma (IFN- $\gamma$ ), which is recommended as prophylactic treatment.

Keywords: Chronic granulomatous disease; Interferongamma; Primary immunodeficiency diseases

\section{Introduction}

Chronic Granulomatous Disease (CGD) is an inherited primary immunodeficiency disease (PIDD) which increases the body's susceptibility to infections caused by certain bacteria and fungi. Granulomas are masses of immune cells that form at sites of infection or inflammation. Chronic granulomatous disease is a disorder that causes the immune system to malfunction, resulting in a form of immunodeficiency. Chronic granulomatous disease (CGD) is a rare, inherited immunodeficiency that affects certain white blood cells.

CGD is a heterogeneous clinical presentation immunodeficiency, whose origin can be autosomal recessive or X-linked [1,2]. In this pathology, both neutrophils and macrophages present a decrease in superoxide generation capacity, leading to failures in the synthesis cascades of reactive oxygen species (ROS) and, consequently, in the antimicrobial activity of these phagocytes [3]. One of the main stimuli that induce the activation of ROS synthesizing enzymes is IFN- $\gamma$, a cytokine that potentiates the transcription of genes responsible for the synthesis of this enzyme complex [3]. One of the characteristic findings of CGD is the formation of granulomas [1]. Clinically, the diagnostic hypothesis is inferred from multiple and recurrent infections by catalase-positive microorganisms, in addition to spores and fungal hyphae $[1,3,4]$. Most individuals affected by CGD are diagnosed before the age of five, with confirmation of the disease occurring between two and three years old on average [5]. The most commonly affected organs include the brain, lungs, liver, spleen, and gastrointestinal tract [1]. For diagnostic validation, complementary tests such as direct measurement of superoxide production, reduction of ferricytochrome C, chemiluminescence, NBT reduction, and dihydrorhodamine oxidation test (DHR) are required [4,5]. The first line of treatment currently recommended is based on the administration of sulfamethoxazole-trimethoprim (SMX-TMP), itraconazole, and IFN- $\gamma[1,3]$. We want to report our experience of an atypical case of 8 years-old with CGD.

\section{Case Report}

A patient named R.S., male, 8 years old, has a history of recurrent respiratory infections - severe bronchopneumonia, requiring thoracic drainage and admission to the ICU since the age of three. In January 2015, at the age of five, he was admitted to a local hospital for suspected pulmonary Tuberculosis (TB), and the diagnosis was confirmed by biopsy during hospitalization. After hospitalization, a respiratory evaluation was performed through radiological exams, which indicated chronic pneumopathy. He was then submitted to 
standard treatment preconized by SUS (rifampicin, isoniazid, pyrazinamide, and ethambutol). In December of the same year, he had a routine consultation with a pulmonologist, and there was an impression of recurrent TB, due to clinical and radiological worsening. He underwent the complete treatment with anti-TB drugs once again, until June 2016. During the treatment, in May 2016, the hypothesis of immunodeficiency was put forward, based on the clinical history. A DHR phagocytosis test was performed, in which the diagnosis of CGD was confirmed, through the comparison between control $(98.8 \%)$ and patient results $(6.7 \%)$.

\section{Discussion}

The typical presentation of CGD has lung, skin, lymph nodes, and liver as frequent sites of infection. Besides, as the name suggests, a major feature of this disease is granuloma formation, and these are especially difficult to treat in the gastrointestinal and genitourinary tracts [6]. Since the patient presents only lung lesions, it can be considered as an atypical case. After the diagnosis, itraconazole and SMX-TMP-based prophylactic treatment was initiated. In June 2016, he was admitted to the pediatric ICU, where he had been referred for evaluation of the possibility of a bone marrow transplant. At the time of admission, his respiratory capacity did not tolerate ambient air, and oxygen therapy via nasal catheter was required. The medical team indicated that the transplant should be performed, however, due to an unfavorable financial family context; the procedure wasn't performed, keeping him in outpatient follow-up and prophylactic therapy, similar to the one previously used. In spirometry and gasometric tests performed between 2016 and 2018, there was a gradual improvement in his pulmonary function, considering that his Forced Vital Capacity (FVC) results went from 19\% of expected FVC to $43 \%$ of expected FVC. The radiological exams of the same period showed the persistence of the lesions resulting from the CGD, but to a lesser degree. In 2018, the patient was referred for follow-up in the allergy and immunology sector of the Univates Medical Specialties Outpatient Clinic, in which the possibility of therapy with IFN- $\gamma$ was proposed, aiming at reducing the risk of recurrent infections, due to the patient's current lung condition, and also due to the hypothesis of a bone marrow transplant was ruled out, considering the riskbenefit of such procedure and the family context. Currently, the patient is stable, and still awaits the judicial release of the IFN- $p$, so that he can begin treatment, and continues on a prophylactic regime based on itraconazole and SMX-TMP.

\section{Conclusion}

CGD has a wide range of symptoms, so treatment should be guided based on each patient. In the presented case, the patient is being treated with a prophylactic combination of itraconazole and SMX-TMP. Besides, we are still awaiting judicial clearance by the Brazilian National Health System (SUS) for financial support for IFN- $\gamma$. It is important to emphasize that the emotional accompaniment of the family is an aspect of fundamental importance, both for adherence to the treatment and for the success of the therapy.

\section{Informed Consent}

An informed consent was obtained from our patient's parents for publication of this case report.

\section{Conflict of Interest}

None declared.

\section{References}

1. Chiriaco M, Salfa I, Di Matteo G, Rossi P, Finocchi A (2016) Chronic granulomatous disease: Clinical, molecular, and therapeutic aspects. Pediatr Allergy Immunol. 27: 242-253.

2. Lacerda-Pontes R, Gomes LN, Albuquerque RS, Soeiro-Pereira PV, Condino-Neto A (2019) The extended understanding of chronic granulomatous disease. Curr Opin Pediatr 31: 869-873.

3. Arnold DE, Heimall JR (2017) A review of chronic granulomatous disease. Adv Ther. 34: 2543-2557.

4. Raptaki M, Varela I, Spanou K, Tzanoudaki M, Tantou S, et al. (2013) Chronic granulomatous disease: A 25-year patient registry based on a multistep diagnostic procedure, from the referral center for primary immunodeficiencies in Greece. J Clin Immunol. 33: 1302-1309.

5. Rider NL, Jameson MB, Creech CB (2018); Chronic granulomatous disease: Epidemiology, pathophysiology, and genetic basis of disease. J Pediatric Infect Dis Soc. 7: S2-S5.

6. Vining M, Sharma N, Guill M (2014) Atypical presentation of chronic granulomatous disease with Burkholderia cepacia. BMJ Case Rep. 2014: bcr2013201524. 